\title{
Inhibitory Effects on L-Dopa Oxidation of Tyrosinase by Skin-whitening Agents
}

\author{
Sang-Hoon Jeon, Kyung-Hun Kim, Jong-Uk Koh, and Kwang-Hoon Kong* \\ Department of Chemistry, College of Sciences, Chung-Ang University, Seoul 156-756, Korea. *E-mail: khkong@cau.ac.kr \\ Received February 16, 2005
}

Key Words : Human tyrosinase, Mushroom tyrosinase, Inhibitory effects, L-Dopa oxidation, Skin-whitening agents

Tyrosinase (EC 1.14.18.1) is a copper-containing monooxygenase catalyzing the $o$-hydroxylation of monophenols to the corresponding catechols (monophenolase or cresolase activity), and the oxidation of monophenols to the corresponding $o$-quinones (diphenolase or catecholase activity). ${ }^{1}$ These functions of tyrosinase play an important role in the formation of melanin pigments during melanogenesis. ${ }^{2}$ Melanin production is principally responsible for skin color and plays an important role in prevention of sun-induced skin injury. However, abnormal accumulation of melanin products is responsible for hyperpigmentations including melasma, freckles, and senile lentigines, which could be a serious aesthetic problem.

Recently, a global market demand has developed for skinwhitening agents because some dark-skinned individuals in many countries and races are prefer lighter skin color. ${ }^{3}$ Several tyrosinase inhibitors have been used in the cosmetic industry as skin-whitening agents. ${ }^{4-7}$ Unfortunately, many of these inhibitors have not been demonstrated yet to be clinically efficacious when critically analyzed in carefully controlled studies. Thus, many efforts are still devoted to screening recognized and putative skin-whitening agents. A correct assay method is essential for the development of effective inhibitors of tyrosinase. Until now, the screening and evaluation methods of these skin-whitening agents have been adopted dopa oxidation test by mushroom tyrosinase because of its commercial availability, not human tyrosinase. Moreover, a comparative study on the inhibitory effects of skin-whitening agents by human tyrosinase and mushroom tyrosinase is still undone.

In this study, we investigated inhibitory effects of skinwhitening agents on L-dopa oxidation by human tyrosinase and mushroom tyrosinase, and compared their effects. According to our study described here, L-dopa oxidation activities of human tyrosinase and mushroom tyrosinase show very different inhibitory effects depending upon the nature of skin-whitening agents.

\section{Experimental Section}

Materials. Mushroom tyrosinase, L-3,4-dihydroxyphenylalanine (L-dopa), and kojic acid were obtained from Sigma (St. Louis, USA). DEAE-Sephacel and metal affinity columns were purchased from Pharmacia Biotech (Uppsala, Sweden). All other reagents used were of the highest grade commercially available.

Preparation of human tyrosinase. Human tyrosinase was obtained by expression of a cDNA in E. coli as described previously. ${ }^{8}$ Recombinant human tyrosinase was expressed in E. coli under the control of the tac promoter and purified by a DEAE-Sephacel column and an immobilized metal affinity column. The purified recombinant human tyrosinase appeared as a single protein band corresponding to a molecular weight of $66 \mathrm{kDa}$ on SDS-PAGE, which agreed well with the reported size of the human tyrosinase. ${ }^{9}$ The purified recombinant human tyrosinase was used for the measurement of the inhibitory effects. Unless otherwise indicated, all purification procedures were performed either at $4{ }^{\circ} \mathrm{C}$ or on ice.

Assay of tyrosinase activity. L-Dopa oxidation of tyrosinases was assayed according to the method of Fling et al. ${ }^{10}$ The protein concentration of the enzyme was determined using the BCA assay reagent (Pierce Chemical) using bovine serum albumin as standard. Kinetic parameters such as Michaelis constants $K_{\mathrm{m}}$ and $V_{\max }$ were determined from the equation of the Lineweaver-Burke plot.

Inhibition studies. The inhibitory effects on the activity of the enzyme were measured by preincubating the enzyme with each inhibitor for $2 \mathrm{~min}$ and initiating the reaction by addition of $3 \mathrm{mM}$ L-dopa at $37{ }^{\circ} \mathrm{C}$. The concentration of inhibitor giving $50 \%$ inhibition $\left(\mathrm{IC}_{50}\right)$ was determined from plot of residual activity against inhibitor concentration. ${ }^{11}$ In this study, we determined $\mathrm{IC}_{50}$ values of six inhibitors that were widely used as skin whitening agents.

\section{Results and Discussion}

To determine optimal conditions on L-dopa oxidation of human tyrosinase and mushroom tyrosinase, we investigated the optimum temperature, optimum $\mathrm{pH}$, and the effects of substrate concentration. The optimum temperature on Ldopa oxidation of human tyrosinase was $50^{\circ} \mathrm{C}$ (Fig. 1). The enzyme activity rapidly decreased above $55^{\circ} \mathrm{C}$. On the other hand, the optimum temperature on L-dopa oxidation of mushroom tyrosinase was $40{ }^{\circ} \mathrm{C}$. Therefore, human tyrosinase was more stable than mushroom tyrosinase.

The effect of $\mathrm{pH}$ on L-dopa oxidation of tyrosinase is shown in Figure 2. The optimum $\mathrm{pH}$ of human tyrosinase was 7.5. The enzyme showed less than $30 \%$ of its maximum activity below $\mathrm{pH}$ 6.5. On the other hand, the optimum $\mathrm{pH}$ 


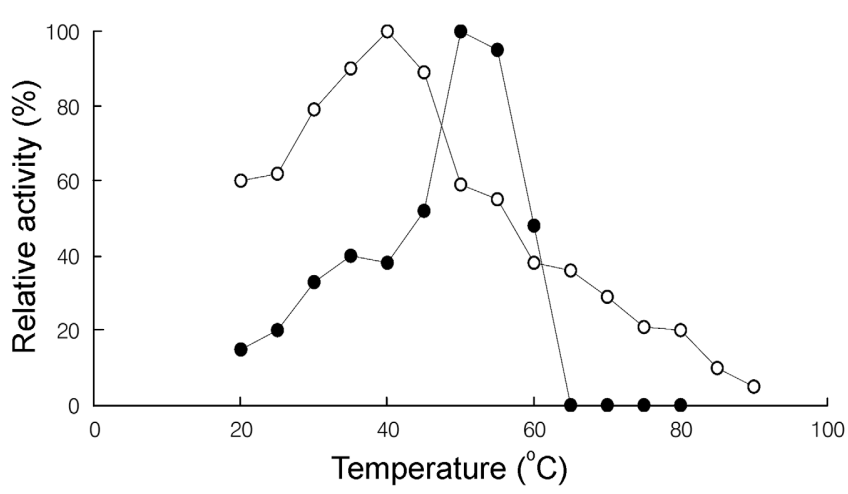

Figure 1. Optimum temperature for the L-dopa oxidation of the human tyrosinase $(\bullet)$ and the mushroom tyrosinase $(\bigcirc)$. In order to understand the effect of temperature, various temperature range, from $30{ }^{\circ} \mathrm{C}$ to $90{ }^{\circ} \mathrm{C}$, of reaction mixture was investigated. The maximum activity was expressed as $100 \%$.

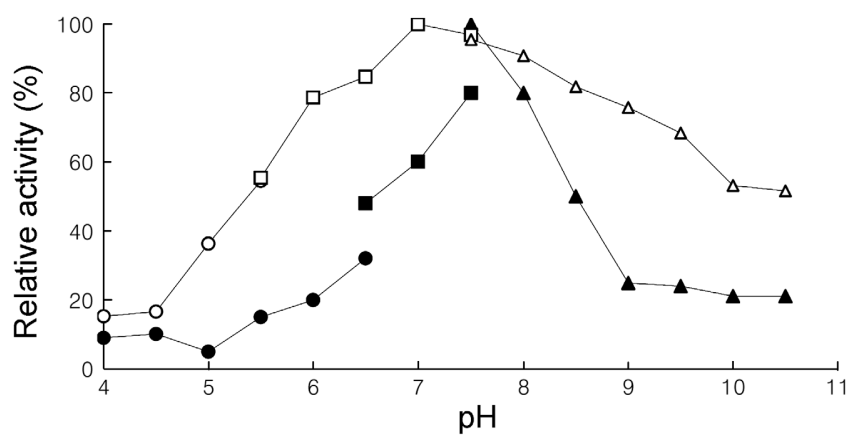

Figure 2. Optimum $\mathrm{pH}$ for the L-dopa oxidation of the human tyrosinase ( $\bullet$ ) and the mushroom tyrosinase $(\bigcirc)$. Optimum $\mathrm{pH}$ of the enzyme was determined by using the following buffers $(100$ $\mathrm{mM}$ ) at the indicated $\mathrm{pH}$ : sodium acetate buffer, from 4.0 to 6.5 $(\bullet-\bigcirc)$; potassium phosphate buffer, from 6.5 to $7.5(\boldsymbol{\square}-\square)$; tris$\mathrm{HCl}$ buffer, from 7.5 to $10.5(\Delta-\triangle)$. The maximum activity was expressed as $100 \%$.

of mushroom tyrosinase was 7.0.

Kinetic parameters were determined from the equation of the Lineweaver-Burke plot. The $K_{\mathrm{m}}$ value for L-dopa of human tyrosinase was $0.31 \mathrm{mM}$ (Fig. 3(a)). This value was similar to that of the tyrosinase from melanoma cell, which was reported to be $0.4 \mathrm{mM}$ for L-dopa. ${ }^{12}$ On the other hand, the $K_{\mathrm{m}}$ value for L-dopa of mushroom tyrosinase was 1.88 $\mathrm{mM}$ (Fig. 3(b)). These data indicate a higher affinity of human tyrosinase for L-dopa.

To evaluate inhibitory effects of skin-whitening agents on (a)

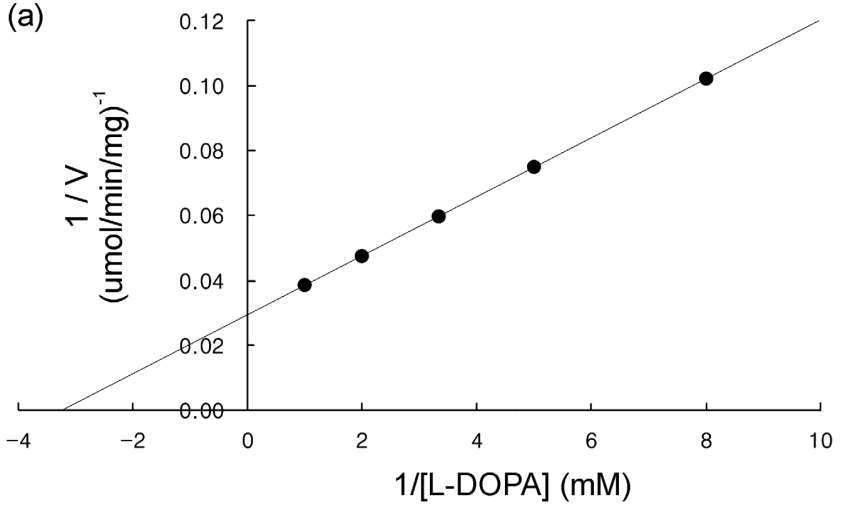

(b)

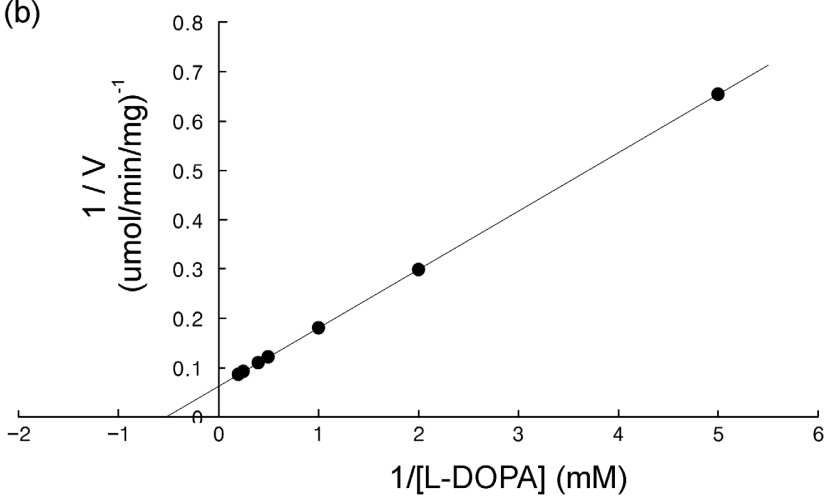

Figure 3. Lineweaver-Burk plot of the human tyrosinase (a) and mushroom tyrosinase (b) toward L-dopa.

L-dopa oxidation of tyrosinase, $\mathrm{IC}_{50}$ values were determined from plot of residual activity against inhibitor concentration (Table 1). Inhibitory effect of arbutin and ethyl ascorbyl ether against human tyrosinase activity was better than that against mushroom tyrosinase activity. On the other hand, inhibitory effect of kojic acid and oil soluble licorice extract against mushroom tyrosinase activity was better than that against human tyrosinase activity. Many researchers have demonstrated that tyrosinase, in the presence of ascorbic acid, develops a lag period prior to any change in absorbance. ${ }^{13}$ Thus, in this study, inhibitory effect of L-ascorbic acid against tyrosinase activity was determined using the minimum concentration of inhibitors induced lag period. The lag period appeared in L-dopa oxidation of human tyrosinase when L-ascorbic acid concentration was above $0.1 \mathrm{mM}$ (Table 1). On the other hand, that in mushroom tyrosinase appeared when L-ascorbic acid concentration was

Table 1. Inhibition effects ( $\mathrm{IC}_{50}$ values) of skin-whitening agents on L-dopa oxidation of the human tyrosinase and the mushroom tyrosinase

\begin{tabular}{ccc}
\hline Inhibitors & Human Tyrosinase & Mushroom Tyrosinase \\
\hline Arbutin & $4.33 \pm 0.08 \mathrm{mM}$ & $10.58 \pm 0.42 \mathrm{mM}$ \\
Kojic acid & $2.73 \pm 0.02 \mathrm{mM}$ & $0.11 \pm 0.01 \mathrm{mM}$ \\
L-Ascorbic acid & $\geq 0.1 \mathrm{mM}$ (lag period) & $\geq 0.02 \mathrm{mM}$ (lag period) \\
Ethyl ascorbyl ether & $16.65 \pm 1.26 \mathrm{mM}$ & $29.69 \pm 2.01 \mathrm{mM}$ \\
Magnesium-L-ascorbyl 2-phosphate & $\geq 5 \mathrm{mM}($ lag period) & $\geq 5 \mathrm{mM}($ lag period) \\
Oil soluble licorice extract & $3.69 \pm 0.10 \mu \mathrm{g} / \mu \mathrm{L}$ & $1.28 \pm 0.12 \mu \mathrm{g} / \mu \mathrm{L}$ \\
\hline
\end{tabular}


above $0.02 \mathrm{mM}$. Therefore, inhibitory effect of L-ascorbic acid against mushroom tyrosinase activity was better than that against human tyrosinase activity. In addition, as the concentration of ascorbic acid increased, the lag period grew longer. Our result also indicated that human tyrosinase and mushroom tyrosinase activities had the lag period when magnesium-L-ascorbyl 2-phosphate concentration reached at $5 \mathrm{mM}$. By comparison of minimum concentrations of inhibitors induced the lag period, L-ascorbic acid was more effective inhibitor than magnesium-L-ascorbyl 2-phosphate.

These rather striking differences in the inhibitory effects of human tyrosinase and mushroom tyrosinase are most likely to be correlated with structural differences. The BLAST program analysis showed that the amino acid sequence of human tyrosinase ${ }^{14}$ identified only $23 \%$ with that of mushroom tyrosinase (Gene bank accession No., O42713). Moreover, we showed in this study that optimum $\mathrm{pH}$, optimum temperature, $\mathrm{IC}_{50}$ values, and kinetic parameter of human tyrosinase are different from those of mushroom tyrosinase. Therefore, it is important to use human tyrosinase for the screening and the evaluation of skin-whitening agents.

Acknowledgements. This research was supported by the
Chung-Ang University Research Grants in 2005.

\section{References}

1. Briganti, S.; Camera, E.; Picardo, M. Pigment Cell Res. 2003, 16, 101.

2. Rescigno, A.; Sollai, F.; Pisu, B.; Rinaldi, A.; Sanjust, E. $J$ Enzym. Inhib. Med. Chem. 2002, 17, 207.

3. Dooley, T. P. J. Dermatol. Treat. 1997, 8, 275.

4. Guevara, I. L.; Pandya, A. G. Int. J. Dermatol. 2001, 40, 212.

5. Nguyen, Q. H.; Bui, T. P. Int. J. Dermatol. 1995, 34, 75.

6. Okan, G.; Baykal, C. J. Eur. Acad. Dermatol. Venereol. 1999, 13, 218.

7. Maeda, K.; Fukuda, M. J. Pharmacol. Exp. Ther. 1996, $276,765$.

8. Kong, K. H.; Park, S. Y.; Hong, M. P.; Cho, S. H. Comp. Biochem. Physiol. 2000, 125, 563 .

9. Wittbjer, A.; Dahlback, B.; Odh, G.; Rosengren, A. M.; Rosengren, E.; Rorsman, H. Acta Derm. Venereol. (Stockh) 1989, $69,125$.

10. Fling, M.; Horowitz, N. H.; Henemann, S. F. J. Biol. Chem. 1963 , 238, 2045

11. Park, H. J.; Koh, J. U.; Ahn, S. Y.; Kong, K. H. Bull. Korean Chem. Soc. 2005, 26, 433.

12. Kang, S. J.; Choi, J. D. Korean Biochem. J. 1993, 26, 632.

13. Zhou, P.; Smith, N. L.; Lee, C. Y. J. Agric. Food Chem. 1993, 41, 532 .

14. Kwon, B. S.; Haq, A. K.; Pomerantz, S. H.; Halaban, R. Proc. Natl. Acad. Sci. U.S.A. 1987, 84, 7473. 